\title{
HBIM GENERATION: EXTENDING GEOMETRIC PRIMITIVES AND BIM MODELLING TOOLS FOR HERITAGE STRUCTURES AND COMPLEX VAULTED SYSTEMS
}

\author{
F. Banfi \\ Dept. of Architecture, Build Environment and Construction Engineering, Politecnico di Milano, Milan, Italy - \\ fabrizio.banfi@polimi.it
}

Commission II, WG II/8

KEYWORDS: Geometric Primitives, 3D drawing, Scan-to-BIM, Vault modelling, Grade of generation (GOG), Modelling automation, Historic Building Information Modelling (HBIM)

\begin{abstract}
:
Today, the generation of smart models and digital archives able to accompany the management of architectural assets through the integration of advanced 3D survey techniques and Historic Building Information Modelling (HBIM) becomes an irremissible added value for the management of building life cycle. New international BIM standards are trying to give some guidelines to this new digital tool, which has demonstrated a wide range of potential applications in digital heritage, structural simulation, generative design, site management, restoration and daily maintenance activities. To manage a proper 3D reconstruction from point clouds, also known as Scan-to-BIM process, BIM experts typically use 3D application developed for the management of new buildings, which make it difficult to generate complex objects such as historic vaults and irregular and damaged walls. For these reasons, this study places the heart of its research in the integration of generative modelling in BIM application, proposing new Scan-to-BIM modelling requirements for complex vaulted systems and a novel add-in ( a set of new functionalities for Autodesk Revit) able to improve the generation of complex elements from point cloud data. In particular, new modelling requirements such as novel grades of generation (GOG) and accuracy (GOA) are integrated within the Revit's structure in order to improve the level of information connected to HBIM and consequently to support the decision making of restoration and rehabilitation projects of complex heritage structures.
\end{abstract}

\section{INTRODUCTION: BACKGROUND AND CONTEXT}

The digital era in which we live is characterised by a continuous evolution of knowledge, working methods and updating processes that we apply in our daily work. The fields of information communication technology (ICT) and system programming mainly increased the market efforts, thus supporting current needs of professionals involved simultaneously (co-working) in the most diverse procedures and disciplines (Georgopoulos, 2018) (Ioannides \& Quak, 2014).

On the other hand, the frantic and continuous development of new application and software requires a holistic ability to update own digital workflows both from a theoretical and technical point of view. In this context, countries like the United States, United Kingdom, Scandinavia, Germany, Singapore, France, China, Taiwan and South Korea are providing excellent solutions for improvements in productivity and cost-savings through the use of BIM in architecture, engineering and construction (AEC) sector. Europe is also developing world-renowned skills and HBIM methods based on the use of new technologies to preserve, manage and leverage value from cultural heritage (CH) (Dore \& Murphy, 2017). Some of them have been introduced in their national regulations, obtaining impressive results for the conservation of heritage buildings. Furthermore, it was found that, whatever its genesis, HBIM needs new modelling requirements, new IT knowledge, constant maintenance and management to be retained, transmitted to different users and sustainably applied by professionals involved in the process, both beginners and advanced BIM users (Della Torre, 2015),(Fai \& Sydor, 2013). For this reason, it should be taken into account that BIM usage is exceptionally efficient when the user can control the construction logic of building in a virtual environment by adjusting every architectural element, linking and mapping a vast amount of data and maintaining the bidirectional relationship between 3D objects and information. For this reason, the following research proposes novel scan-to-BIM modelling requirements and an add-in for Autodesk Revit in order to simply the generative process of historical vaults using point cloud data.

\section{PROS AND CONS IN HBIM GENERATION: BIM SOFTWARE AND FREE-FORM MODELLING}

The main problems encountered for the generation of an asfound model (AF-BIM) and HBIM models can be summarised in two distinct modelling families: BIM and free-form modelling software.

BIM applications allow users to create a building and its components in a digital environment, annotate the model with 2D drafting elements, and access building information from the building model's database. They include categories of predefined objects ('families' in BIM terminology) that describe simple building components such as walls, floors, doors, windows, ceilings and roofs. Each item is adjustable in its subcomponents, and the type of each element can be edited through the use of settable parameters (before and after).

This ability allows users to change the features of each element after their generation through tools that can update physical quantities such as height, width, scale and their physical and thermal characteristics (i.g. types of materials and wall stratigraphy). On the other hand, the main BIM applications are characterised by a limited number of modelling tools and libraries of objects that geometrically represent simplified architectural elements.

Therefore, the lack of modelling tools able to automatically transform point clouds in HBIM objects prevents the generation of complex elements, as well as the information mapping and information sharing during the building life cycle (BLC). 
Free-form modelling software, on the contrary, is based-on generative modelling which focuses on producing a mathematically precise representation of curves and freeform surfaces. The most commonly used free-form modellers are Mc Nell Rhinoceros, Autodesk Inventor, Autodesk Autocad, Solidworks, and Blender. Most of them are characterised by NURBS-based generative modelling functions that allow the generation of 3D geometric entities able to interpret any form, even the most complex. Through a large number of advanced modelling tools, it is possible to create surfaces and solids starting from geometric primitives such as points, lines and splines. Accordingly, a proper Scan-to-BIM model should be based on the use of modelling requirements able to transform geometric primitives such as 3D scans, points and lines in smart BIM objects properly. This transition requires high knowledge of different modelling techniques, new exchange formats and modelling solutions in order to create 'informative' models able to support the digital management of heritage buildings holistically. On the other hand, NURBS models cannot be considered real BIM, but just simple entities such as surfaces and solids. From a semantic point of view, these 3D entities lack the capacity to seamlessly connect, share, and update different type of contents in real time. This parametric feature remains a prerogative of BIM objects that allows users to create digital repository, schedules and databases automatically.

Figure 1 shows the main gaps in the generative process of HBIM models, highlighting the barriers encountered in BIM and free-form modelling software for each phase.

MAIN GAPS IN THE GENERATIVE PROCESS OF HBIM

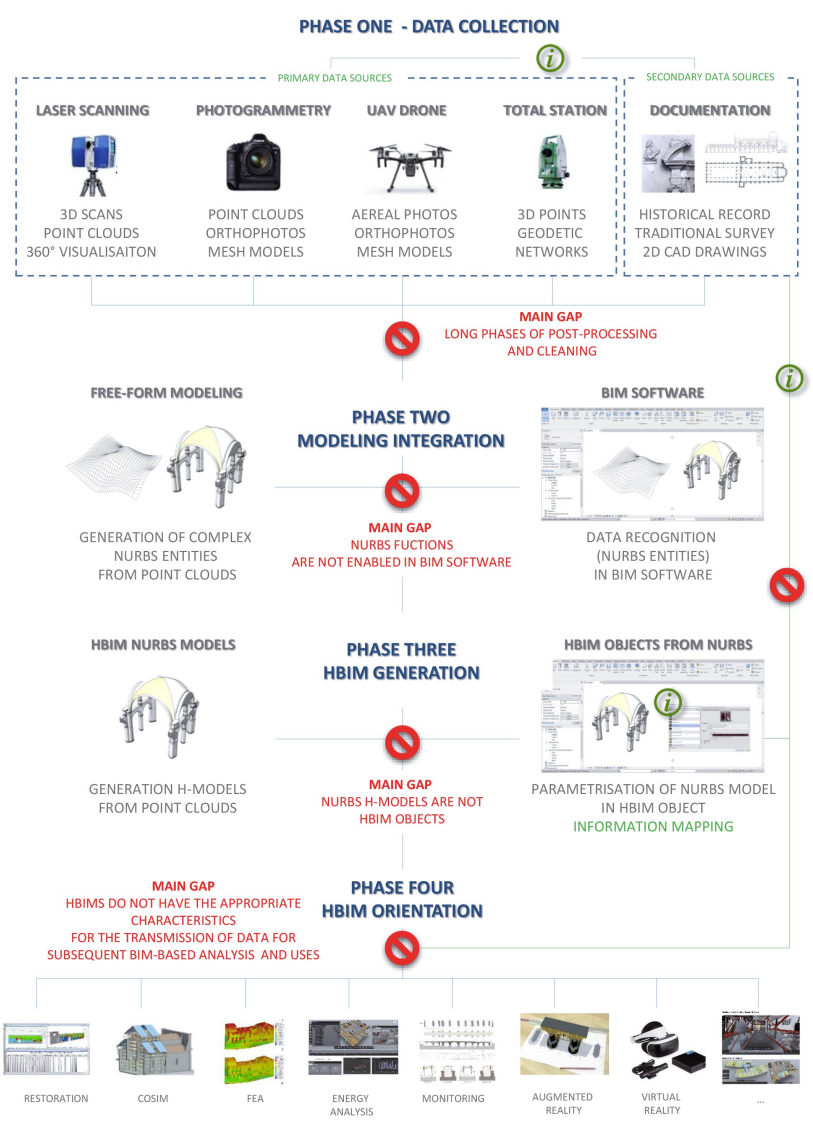

Figure 1. The main gaps in the generative process of HBIM models. The information sharing is hampered due to a lack of modelling tool able to transform point clouds in smart BIM objects (generation) for different type of analysis (orientation).

\section{RELATED WORKS AND RESEARCH OBJECTIVES}

In the last five years, previous studies have shown how novel scan-to-BIM modelling requirements have obtained considerable proper results in different field of application. To lay the foundations of this research, the first study considered is 'BIM orientation: grades of generation and information for a different type of analysis and management process'(Banfi, 2017). It provides for the establishment of a list of approved Scan-to-BIM modelling requirements able to overcome the main modelling barriers in the HBIM field.

In particular, thanks to the development of a novel Modelling Management Information System (MMIS), new grades of generation (GOGs) and accuracy (GOA), it has been possible to improve the level of automation in the Scan-to-BIM process.

Figure 2 summarises the ten GOGs. In order to classify the grade of modelling generation, several modelling tests on the interpolation of 3D survey data have highlighted the lack of advanced modelling tools within BIM applications, leading to new IT developments. The flexibility of the method is based on a free interpretation of the data and a free identification of the points for the automatic or semi-automatic extraction of geometrical primitives useful for the generation of digital models. The application of Non-Uniform Rational BasisSplines (NURBS) interpolation algorithms between outer edges and known points has allowed an automatic generation of complex surfaces with a high number of internal $\mathrm{U}, \mathrm{V}$ subdivisions.

The Ten Grade of Generation in MMIS

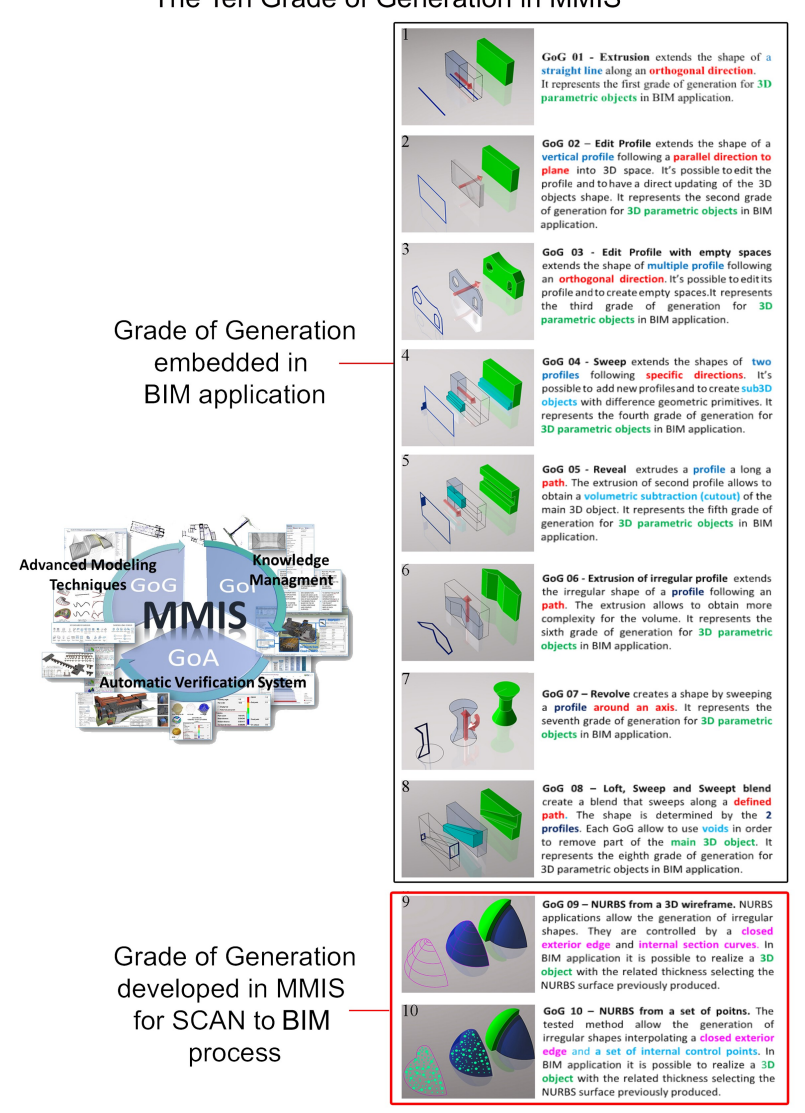

Figure 2. Novel Scan-to-BIM modeming requirements: the ten Grade of Generation.

SOURCE: Banfi, F., 2017: BIM orientation: grades of generation and information for different type of analysis and management process, Int. Arch. Photogramm. Remote Sens. Spatial Inf. Sci., XLII-2/W5, 57-64, doi.org/10.5194/isprsarchives-XLII-2-W5-57-2017 
The ten GOG are divided into two categories:

- the first eight grades of generation correspond to the modelling functions integrated into the BIM application.

- $\quad$ the last two grades (9-10) of generation correspond to two new advanced modelling techniques (slicing and automatic interpolation) capable of transforming dense point clouds into the digital parametric model.

The first eight GOGs consequently clearly explain how a wall can be realised in Autodesk Revit. GOGs defines the techniques and requirements used to improve the quality of the BIM models. The complexity of the built heritage and the generation of irregular walls currently involve the direct insertion of the wall object into the three-dimensional space through the GOG1. Subsequently, through GOG 2,3,4, it is possible to model and increase the level of detail (LOD) of the models.

On the other hand, when the point clouds express volumetric irregularities and complex geometry, to achieve a higher LOD than 300, the use of GOG 9 and GOG 10 is crucial.

GOG 9 is a modelling procedure that summarises the known slicing technique. The traditional process of generating 2D CAD models and drawings is essentially based on the manual extraction of cross-sections from point clouds. The sections, usually used for technical drawings, are used as a generative basis for three-dimensional modelling in the digital space. GOG 9 , instead, bypass $2 \mathrm{D}$ drawings and permits the generation of complex models through the automatic extraction of geometric primitives. GOG 9 is based on the generation of NURBS from 3D wireframe. They are controlled by a closed exterior edge and internal section curves (in pink in figure 3). In BIM application, it is possible to realise a 3D object (in green) with the related thickness selecting the NURBS surface previously produced (in blue). Figure 3 shows the GOG 9 application for some complex vaulted systems of Masegra Castel. The modelling procedure is composed of four steps:

1. Generation of a closed exterior edge

2. Extraction of internal section curve

3. Automatic generation of NURBS surface (50x50 UV)

4. The parametrisation of the NURBS surface in BIM object

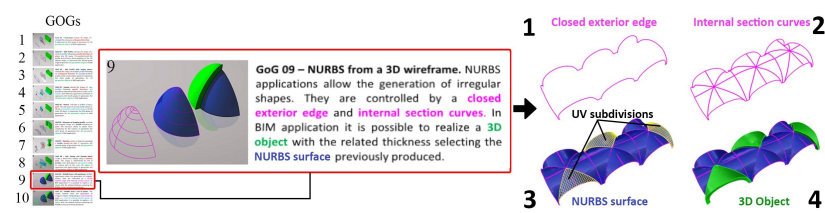

Figure 3. GOG 9: Automatic extraction of proper geometric primitives (1-2) for the generation of the NURBS model (3) and the subsequent automatic generation of complex Scan-to-BIM object (4).

GOG 10 is a three-step modelling procedure that has allowed the reduction of time necessary for the generation of HBIM. The generation of internal curves able to interchange the irregular internal shapes of the irregular elements was substituted with 2 requirements. Geometrics can maintain a grade of accuracy and realize AB-BIM with a LOD 500. The digitalisation of built heritage requires a user-friendly modelling method able to intercept the geometrical and structural anomalies inherited from a millenary historical heritage. For this reason, GOG 10 should be the first requirement for the generation of historical and unique elements. GOG 10 is a modelling procedure able to generate a NURBS from a set of point (Fig.4). The tested method allows the generation of irregular shapes interpolating a closed exterior edge and a set of internal control points. Subsequently, in BIM application, it is possible to realise a 3D object with the related thickness selecting the NURBS surface previously produced.

NURBS interpolation algorithms were able to generate complex surfaces that interpolate a large number of points of the 3D scan. Once the point cloud is converted into the NURBS surface by defining specific exchange formats (.sat,.dwg), it is possible to parameterise the NURBS surface within Autodesk Revit. Figure 4 shows the GOG 10 application on a complex vaulted system. Three steps compose the modelling procedure:

1. Generation of a closed exterior edge

2. Automatic generation of NURBS surface (50x50 UV)

3. The parametrisation of the NURBS surface in BIM object

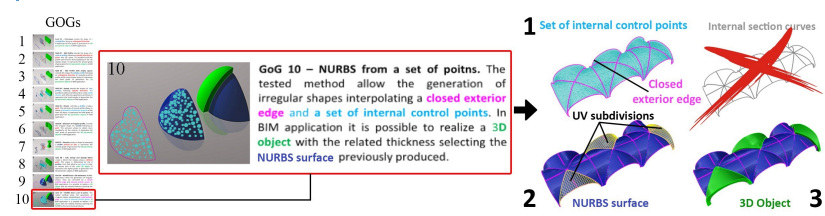

Figure 4. GOG 10 allowed the generation of any vault with a GOA of $1 \mathrm{~mm}$ from the 3D scan (1), the automatic generation of the 3D objects (3) from a NURBS model (2) in Revit.

The first benefit of this integration between NURBS modelling, 3D scans, and BIM software is the automation of the generative procedure for complex elements. This new generative process, based on GOG 9 and GOG 10, has ensured to preserve all the geometric anomalies of each architectural and structural element in HBIM and to link a great quantity of information to every single 3D object for the management of heritage structures over the years.

The second study considered is 'The integration of a Scan-toHBIM process in BIM application: the development of an addin to guide users in Autodesk Revit' (Banfi, 2019). This research shows how it was possible to develop and improve Revit functions and modelling tools thanks to an accurate study of the application programming interface (API). Autodesk, an international leader for digital applications oriented to the construction sector, has made available new exchange formats and generative logics that are less binding than in past decades. In light of this, the availability of shared languages published online in cloud developer platforms (Autodesk Forge and Revit API), has allowed the author to undertake the design of a Revit add-in (Scan-to-BIM facility) with the main aim of supporting Revit users in HBIM generation.

From an operative point of view, IT development involved the use of Revit API docs. Application Programming Interface databases made it possible to identify the procedures available to the programmer, grouped to form a set of specific tools for the accomplishment of a specific task within Autodesk Revit. Thanks to 'My First Plug-in' has been possible to have a self-paced tutorial guide for a smooth introduction to the programming world. This first step is a one-stop shop learning path for users who know Revit but are new to programming and are thinking about taking the plunge. The guide has introduced how to work the Revit .NET API programming language. Autodesk Revit provides a rich and powerful .NET API which can be used to automate repetitive 
tasks, extend the core functionality of Revit in simulation, conceptual design, construction and building management. Revit .NET API allows the programming with any .NET compliant language including VB.NET, C\#, and C++/CLI (Fig.5). The software development toolkit (SDK) provides extensive .NET code samples and documentation to support the developing with the Revit API. Autodesk has made available Revit API enhancements added since the initial release of Revit 2018.

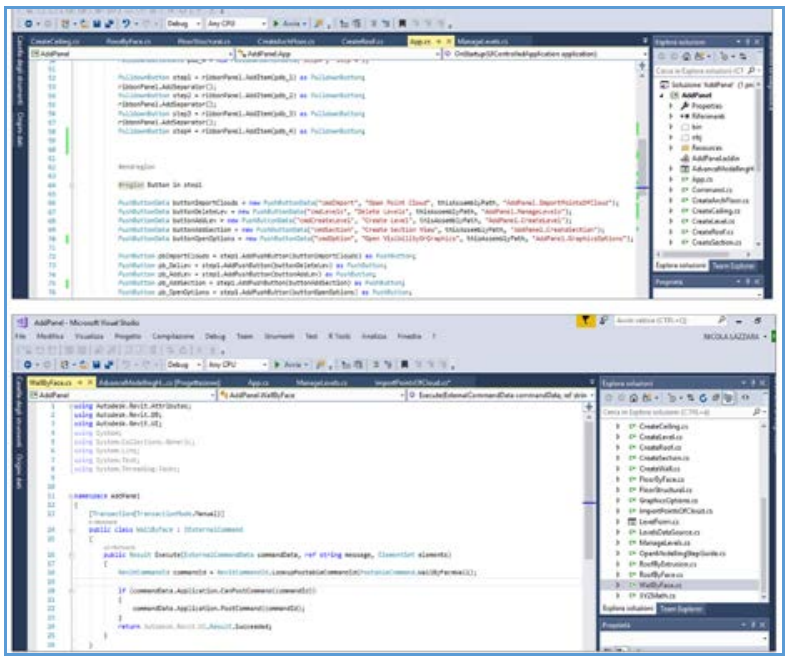

Figure 5. The add-in's development phases in Visual Studio.

The enrichment of 3D holistic 'informative' models with metadata and semantics in $\mathrm{CH}$ has transmitted within the process thanks to the development of four sections (Fig.6):

1. Scan management: to facilitate the import of the 3D scan and to support the setting of Revit project for Scan-to-BIM process;

2. Modelling (GOGs): to integrate GOGs (9 \& 10) in Revit architecture and to guide users in the creation of complex walls and historic elements with generative modelling;

3. Database generation (GOIs): to automate database generation and to enable the addition of new custom parameters;

4. Interoperability levels: to define novel exchange formats and to transfer HBIM model to different BIM-based analysis.

The add-in 'Scan-to-BIM Facility Tool', from an operational point of view, provides the requirements for an advanced modelling able to integrate NURBS geometry into BIM applications. Consequently, this add-in represents the maximum possible result in terms of development oriented to integrate the research process in Autodesk Revit. Add-in facility tool has allowed:

- the 3D scans integration into BIM application through a guided path,

- the reduction of the modelling costs and the production time

- Scaling the process by creating databases for complex historical objects, customizing new parameters

- better BIM orientation thanks to new levels of interoperability for different BIM-based analysis such as computing, BIMcloud, virtual and augmented reality (VR-AR).
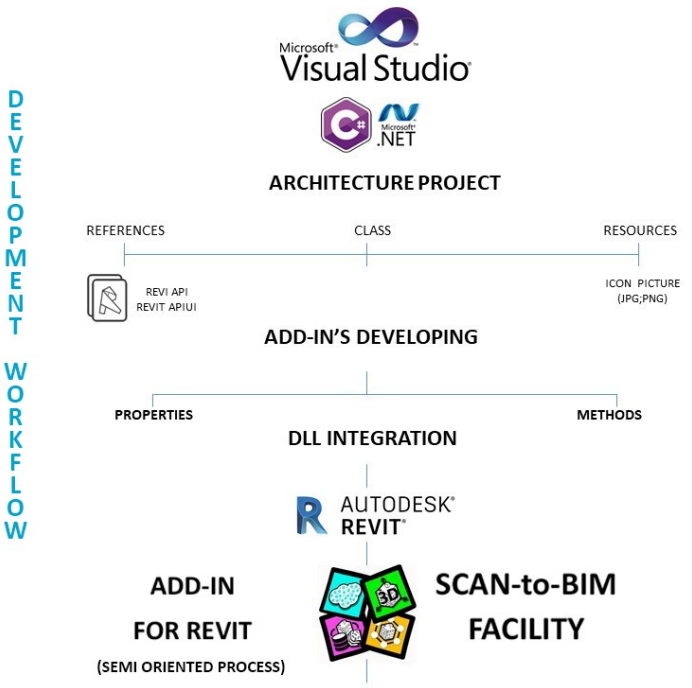

(SEMI ORIENTED PROCESS)

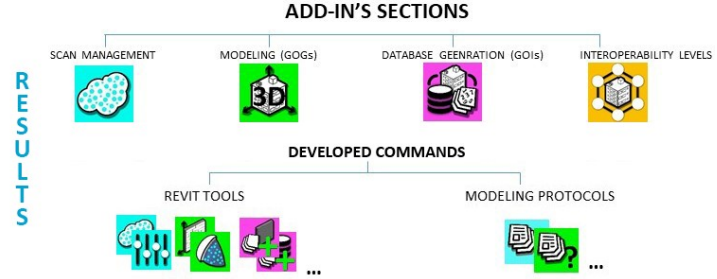

Figure 6. The development process of Scan-to-BIM Facility tool. The add-in allows the integration of the proposed method in BIM application, supporting the user with a guided process.

In these two previous studies, the tangible and intangible values, the uniqueness, richness and irregularity of the shapes of historic buildings such as vaults, arches, walls with variable section, walls with double curvatures, columns and historical decorations represented one of the main challenges in the field of digital reconstruction.

For this reason, the following research has investigated and deepened the main modelling techniques with the aim of identifying the main causes that prevent the spread and adoption of HBIM models and Scan-to-BIM processes for the complex structures. It was found that one of the main causes is given by the absence of new modelling rules capable of transforming survey data (primary data sources) and historical documentation and technical analysis (secondary data sources) into models corresponding to detected reality. The potential of geomatics and the 3D survey techniques opens the door to various forms of digital modelling and information sharing, extending to the field of built heritage (Brumana et al., 2018), (Tucci \& Bonara, 2011).

In this specific context, the importance and the centrality of the user and his knowledge of modelling are the primary sources for the correct management of the process. It was, therefore, necessary to unify efforts, define general rules that allow users to understand the wealth of tangible and non-tangible built heritage values but without creating a 'straitjacket' to modelling techniques.

Once the main barriers in the Scan-to-BIM process have been identified, this research has established new generative requirements for the creation of vault models able to represent the structural elements surveyed with a high grade of accuracy (GOA). The enhancement of information and intrinsic culture of a specific artefact can be improved through the potential of the proposed Scan-to-BIM process. 


\subsection{D Survey cloud-based data collection, post-processing and verification for HBIM generation}

In the recent decade, the development of new technologies played a crucial role during the generative process for heritage models. Currently, market applications of 3D data capture technology such as total station (TS), terrestrial laser scanning (TLS), unmanned aerial vehicle (UAV) and high-resolution cameras for digital photogrammetry is validating to be quick and accurate methods for the proper 3D building survey (Remondino \& Stylianidis, 2016). Thanks to the integrated use of point clouds from laser scanning, photogrammetry and total station it has been possible to achieve a proper base for the digital reconstruction of the research case studies, reducing return site visits and saving a significant amount of time. The LOD of the 3D models and the GOA between point clouds and BIM depended on the quality of the $3 \mathrm{D}$ survey data (point clouds and geodetic network). The main instruments used for the 3D survey of the research case studies are Faro Laser Scanner Focus, Canon EOS-1D X, and Leica TS30.

The fields of application were (Fig.7):

1. the cupola in Saint Vittore in Ciel d'oro in Milan,

2. the sail vault of Basilica of Saint Eustorgio in Milan,

3. the barrel vaults of Azzone Visconti bridge in Lecco,

4. the groin vaults of Masegra Castel in Sondrio

5. the ribbed vaults of Basilica of Saint Ambrose in Milan

6. the umbrella vault of Basilica of Collemaggio in L'Aquila.
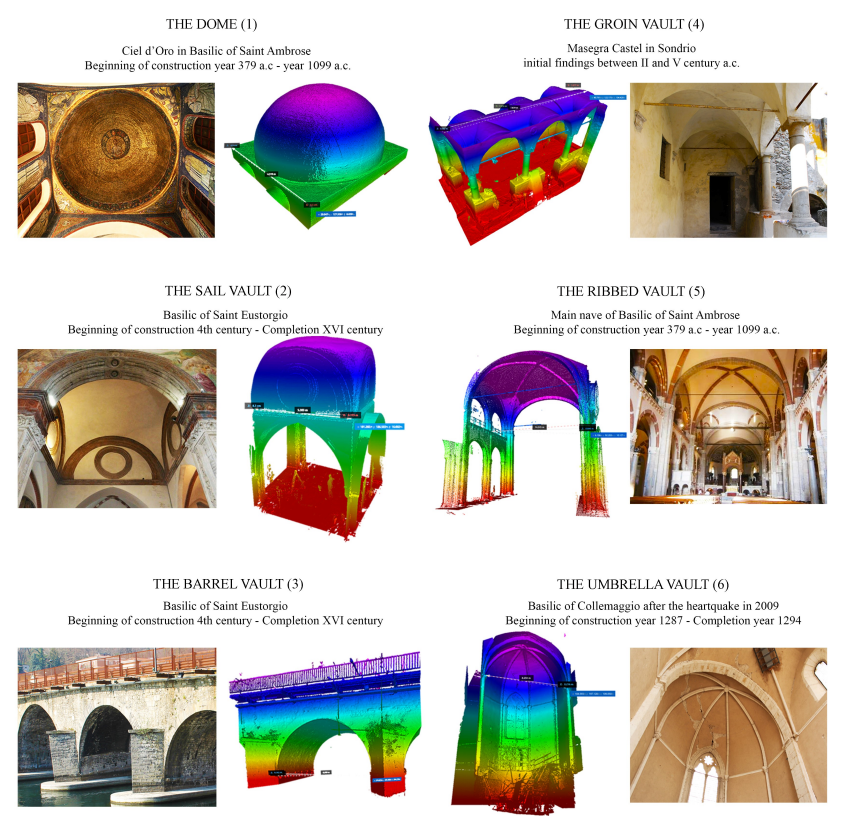

Figure 7. The wide variety of vaulted systems of the research case studies and the related point clouds.

Each case study has brought important results in different fields of application and analysis, transforming the vault model from a three-dimensional representation to a management process.

This study has investigated the geometric generation of various architectural vaults, demonstrating how the concept of parametrization of a NURBS surface can be associated with a BIM object, BIM databases and BIM clouds.

\section{THE ESTABLISHMENT OF SCAN-TO-BIM MODELING REQUIREMENTS FOR COMPLEX VAULTED SYSTEMS}

The tests carried out on the generation of a NURBS surface have highlighted that it is absolutely essential to obtain a wall thickness with the ability to adapt rapidly over time.

The proposed method has found the best solution by integrating the NURBS surface into the Autodesk Revit's mass subcommand. However, the use of geometric primitives requires reference planes in which to draw, not allowing the direct use of point clouds for complex structural objects.

Thanks to the definition of GOG 10 it was possible to use the masse command (Autodesk Revit tool) only for importing the NURBS surface, thus avoiding long modelling phases. Finally, thanks to the 'wall by face tool' it was possible to automatically create the wall thickness of the NURBS surface, thus generating a BIM wall object in all respects, by enabling information mapping and information sharing functions. As a result, it was possible to import a large amount of previously modelled NURBS surfaces into MC Neel Rhinoceros and to avoid modelling in Autodesk Revit for $90 \%$ of the building. Once the parametrization of the surface in Autodesk's Revit was successful, the BIM wall-object became an 'informative' model prepared to accept each type of information.

Thanks to this method, BIM objects are able to interpret the geometry of the point cloud by following the general shape of the structural element and not its sub-modules such as stones and bricks. It was clear that the level of approximation of a very detailed BIM vault couldn't be made up in detail, brick by brick, stone by stone. Furthermore, it was found that the 'granular modelling' of every single constructive element in addition to requiring long generative phases and high costs does not allow to optimise the appropriate association of the information in BIM logic.

The primary objective of simplifying the modelling process required a hierarchical classification of the vaults in order to distinguish the operations necessary for their 3D generation.

The first major distinction made was based on their geometric structure and geometry:

- $\quad$ vaults with a single curved surface (dome, sail, barrel) were placed in the group of simple vaults,

- while vaults formed by a combination of two or more simple vaults (groin, umbrella, ribbed) have been brought together in the complex vaults group.

In summary, the research objectives applied to these six Italian research case studies were:

- To improve the utility of HBIM models through the use of novel scan-to-BIM modelling requirements, managing the complexity of complex vaulted systems with high-grade accuracy (GOA);

- Developing best practices for recording, documentation and information management of complex structures.

- To show the research impact and to prove the economic benefits of the proposed IT development for the generation of complex vaulted systems.

- To show the scalability and applicability of the proposed method for any type of complex architectural and structural elements such as damaged walls, curved walls, arches and archaeological sites. 


\subsection{GOG 10 for different type of vaulted systems}

The Dome (traditional modelling process) represents the most technically significant solution to cover an area without intermediate support structures, with minimal material consumption. The tracking of its structural building blocks (stone or bricks) perfectly responds to the compressions (second oriented meridians), while the joints between each block ensure the resistance of the parallel. The structural problem, for the most part, must ensure structural integrity that can prevent traces in the parallel. This case study deepens a constructive historical technique that can overcome the structural problems of the dome typology, lightening the whole system with a particular construction technique. From a generative point of view, the domes are characterised by a central symmetry or/and by the rotation of a profile around a vertical axis.

A hemispherical cap constitutes a simpler example where the generating curve can generate a hemisphere through a rotation. Essentially, the dome is an arch that is extruded into the third dimension, whereas a dome is an arch revolved around its vertical axis. Often the domes rest on a drum with a prismatic or cylindrical shape, which rests on the basic structures; the drum, in addition to giving greater visibility to a higher dome, serves to improve the natural illumination of the underlying environment by inserting windows. To connect a square base with an octagonal drum, plumes are inserted. Other forms of natural lighting, which connote the general shape of the dome, are lateral openings called eyes or bricks placed at the top of the structure. This particular type of vault is almost always characterized by a very regular shape as well as its generative modelling process based on a great number of modelling steps unable to intercept the complexity of irregular shapes from point clouds. Figure 8 shows the steps required for the generation of a dome with the traditional process.

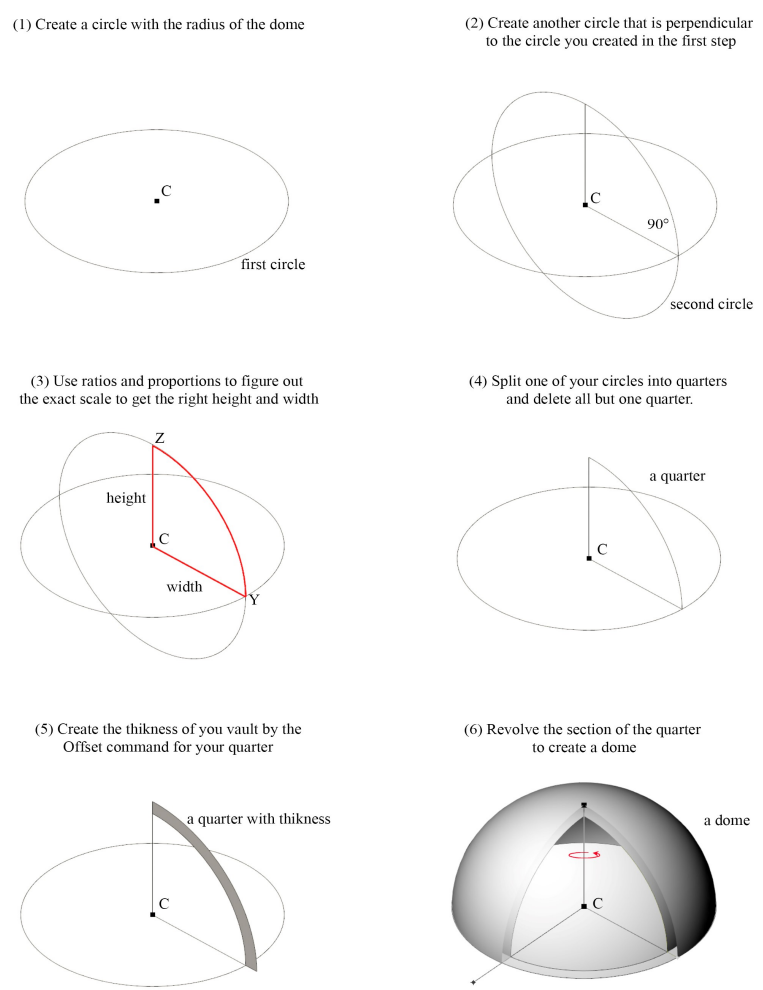

Figure 8. The traditional process: 'how to model a dome'. Each step is not oriented to intercept the real shape of the 3D scans.
The Dome by GOG 10 - The San Vittore chapel in Ciel'oro was built in the first half of the 4th century and is one of the most precious testimonies of Christian Milan. It was used as a burial of Saint Satiro and Saint Vittore martyr.

The building features are a trapezoidal plant with slightly spaced hips and a small apse, covered by a golden eyebrow of Oriental inspiration.

Between the actual dome and the perimeter is inserted the cylindrical drum that can connect the semi-spherical shape to the perimeter walls that support the structure.

Thanks to the laser scanner and photogrammetry, it was possible to detect the structural irregularities of the dome and to emphasize the constructive technique identified by the restoration of the years 1982-85.

In particular, the structure is made up of solid tubules disposed of in concentric circles with decreasing diameter, linked with a magnesium lime mortar with gravel and sand. Each tubule has a cylindrical shape with a conical appendix (8-9 cm long) that is inserted into the cavity of the next tubule (total length $\mathrm{cm}$ 20-23 $\mathrm{cm}$; diameter of $\mathrm{cm}$ 5,5-7; appendix length 8-9 cm).

Thanks to GOG 10 based on the NURBS interpolation of the detected points and the determination of a B-spline (the base of the vault), it was possible to connect these types of information to the Scan-to BIM model of the dome.

Once realised the subsequent parameterization in Autodesk Revit, it was possible to link the constructive technique, to define the vault's stratigraphy and to associate different types of information (Fig.9). The parameters linked to the vault model allow users to figure out the complexity of a unique structure from a morphological point of view, highlighting the importance of generative modelling aimed at making explicit the unique construction technique used.
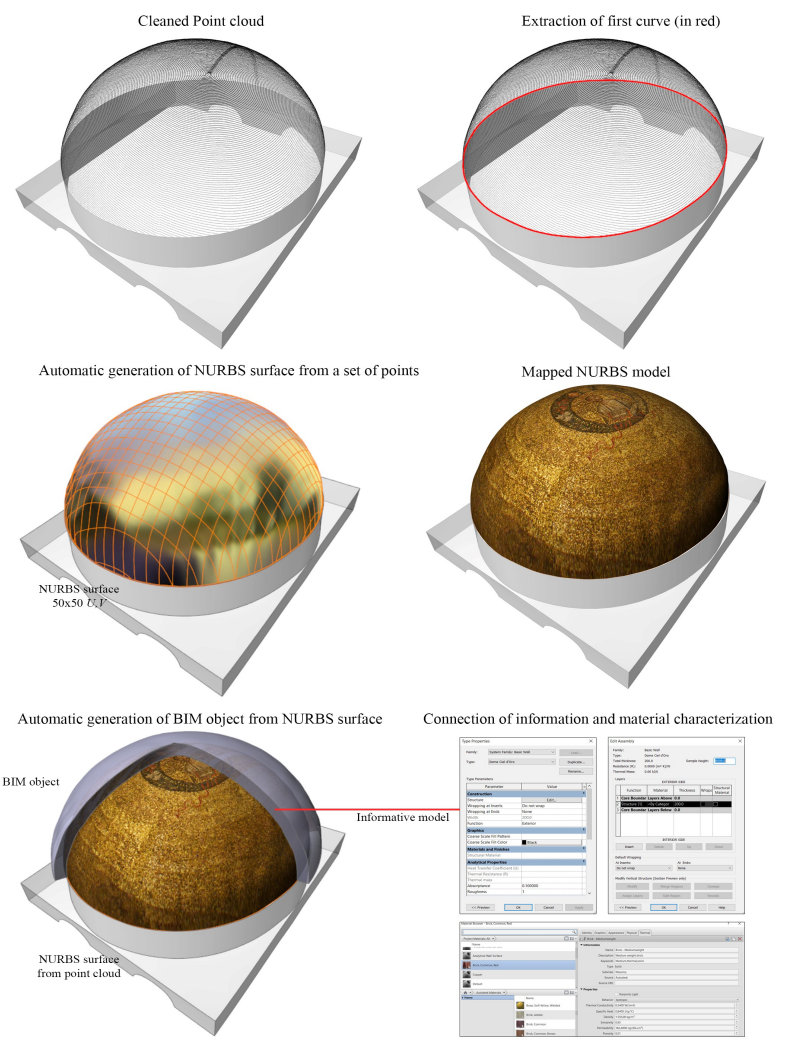

Figure 9. The research method: 'how to model a dome by GOG 10 '. The modelling procedures bridge the gap between NURBS modelling and BIM applications. 
The sail vault (traditional modelling process) is a type of architectural cover similar to a square-shaped dome.

Its structure is semi-spherical and sectioned by vertical planes, through the walls or supporting pillars. The sail vault is therefore delimited by arches resting on the pillars. If it is sewn with a tangent horizontal plane at the arches, the vault is divided into a shell and four spherical pendentives. Pendentives are created to connect a polygonal base to a dome. This type of simple vault, in descriptive geometry, is to indicate a common two solid part formed by:

- a spherical shell characterized by a rotation axis (vertical line),

- a generator meridian (conic arc) and a parallel conductor (circumference).

Present in the oriental as in the western architecture the domes and the sail vaults assume a clear symbolic function. For this reason, as well as for the often considerable dimensions, they stand out from the ordinary structures, incorporated in the buildings and destined to enrich the interior spaces of the buildings with their own shapes.

The first step of the typological metamorphosis passes through the structure of the sail. It is in this way that the problem of the architectural connection between the square and the circular shape is solved. If you remove the cap, in fact, there are only four corner spiers, which make up the ideal base for a cylindrical drum. The transformation of the dome into a sail is therefore obtained by sectioning the circular structure: figure 10 summarises the traditional process for a sail vault.
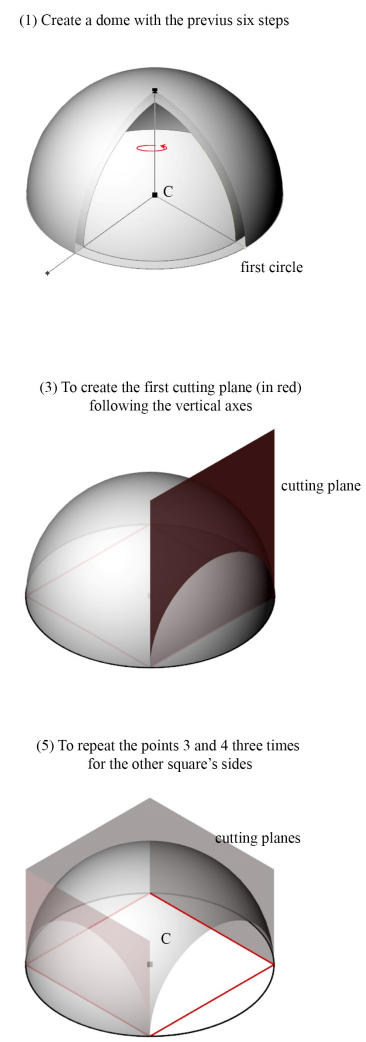

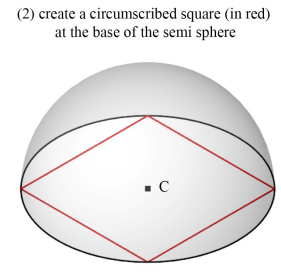

second circle
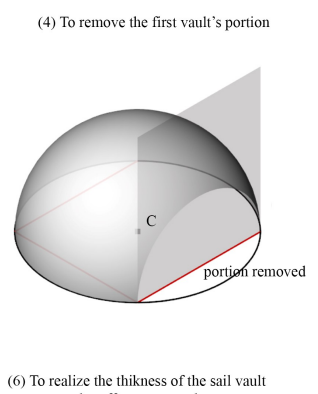
by offset command

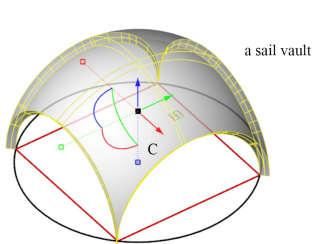

Figure 10. The traditional process: 'how to model a sail vault'. The traditional process requires a great number of modelling steps based on simplified geometric primitives. Each step is not oriented to intercept the real shape of the point clouds characterised by geometric irregularities.
The sail vault by GOG 10 - The research method has been able to avoid the manual generation of various geometric primitives and cutting planes useful for three-dimensional creation. The generation of the surface of Saint Eustorgio's vault in Milan has highlighted the constructive technique of scrap irregularity. It is more complex than the other types of simple vaults because it required the realization of several entities in the digital environment. The construction technique required the first type of cent used to create the central part of the vault that follows the intrados of the semi-sphere, the second typology instead, served for the creation of the four lateral arches.

The bricks were ideally arranged parallel to the basic circular line of the original sphere and cut from a vertical plane of the sharp arch. The modelling foresees the extraction of four arches (B-spline curves) from the point cloud and the direct interpolation of the points of the semispherical surface, significantly lowering the production times. The management of the shape is simplified thank to the automatic NURBS surface generation. As for the dome, the NURBS interpolation has allowed the reduction of the interpreted errors by the user and the automatic recognition of the surface produced in the BIM application. Once the NURBS surface was imported into Autodesk Revit it was possible to automatically transform the surface into a BIM object (Fig.11). The transformation of the NURBS surface to an information model has allowed intruding into articulated digital models, new complex structures that previously could not be created with the basic tools of BIM applications.
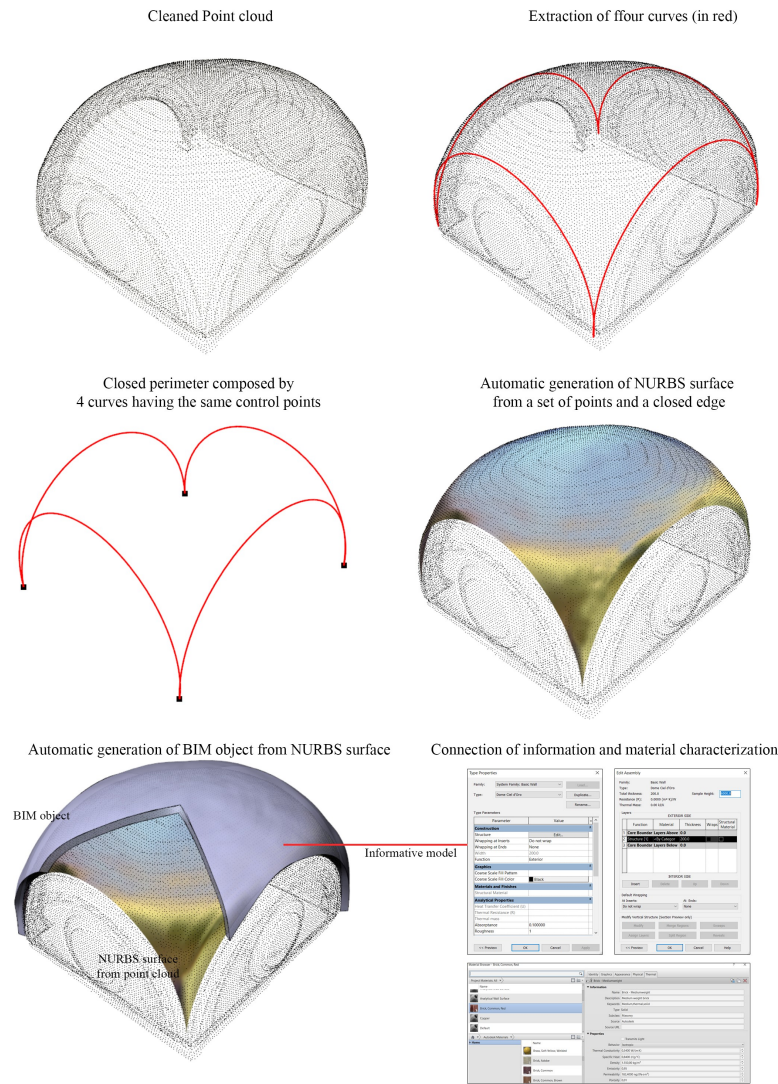

Figure 11. The research method: 'how to model a sail vault by GOG 10 '. Thanks to GOG 10 , it is possible to extract the 3D edge from cleaned point clouds and to create a complex vault automatically, corresponding exactly to the detected reality. 
The Barrel Vault (traditional modelling process) is considered an extruded arch along a well-defined direction. One problem related to this type of simple vault was the generation of pressure on the lower walls of the structure. Typically, due to the heavy arches, an outward force is created in the lower portions which lead to the collapse of the vault. For this reason, during the centuries, thicker walls were made in order to absorb the loads and as the vault's length of increased the method became less advantageous. The traditional construction technique handed down through the centuries requires the creation of a stable work plan to favour both the correct execution of the structure and the protection of the general safety conditions of the operators. The plan is realised in consideration the height from the base below (generally above 2 $\mathrm{m}$ ) and the need to work in length, according to the direction of development of the vault, with the progressive displacement of the rib (Fig. 12). According to the traditional generative procedure, references plane and the arches allow the creation of the barrel vault. On the other hand, these geometric primitives do not lead to embedding specific geometric irregularities localised in the internal portion of the structure. In recent years, most of BIM application has implemented specific modelling tools in order to create more detailed barrel vaults, trying to simplify the generation of the structure with the definition of reference planes and cutting cross-sections.

The slicing technique has been the only one capable of intercepting the complexities and irregularities in the surface of the extrados, but asking for the long manual procedure of the point cloud segmentation.

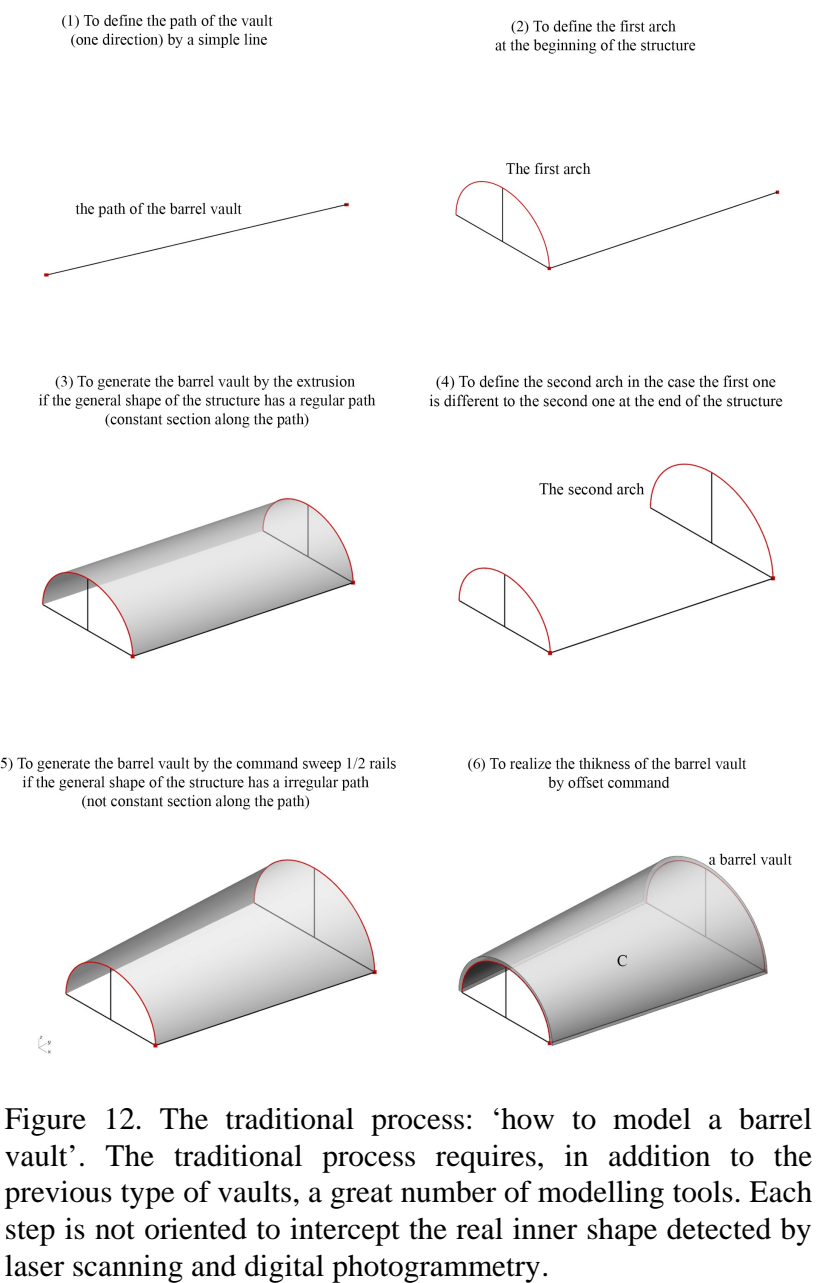

The Barrel Vault by GOG 10 - Thanks to the accuracy of the laser and photogrammetric survey, it was possible to identify the construction technique for the realization of every single structural element. In addition, the detection of the thickness of the bridge holes, the irregular plane of the tide tax, and the evolution of the upper arch, allowed to define the geometric boundaries of the used centring. The case study presented circumscribes one of the most irregular arches of the Azzone Visconti bridge in Lecco.

The bridge has been subject to consolidation, maintenance and restoration work in recent years. Structural surveys have provided for use of the GOG 10, avoiding manual segmentation of the point cloud, and minimizing manual procedure at the same time.

In particular, the extraction of two geometric primitives corresponding to the two closing arches of the vault. Structural irregularities and complex geometries were evident on both sides of the bridge (north and south).

The proposed Scan-to-BIM process has essentially applied the process based on the NURBS interpolation of the two types of previous vaults but with the difference in the number of geometric primitives used. Thanks to the scan's resolution have been created each stone of the main arches and the related stone's thickness from the intrados of the vault. Finally, thanks to the definition of scaffold's holes have been possible to propose a hypothesis of the same. Lines construction intercepted the path and the size of holes used for the thickness definition of the wooden beams (Fig. 13).
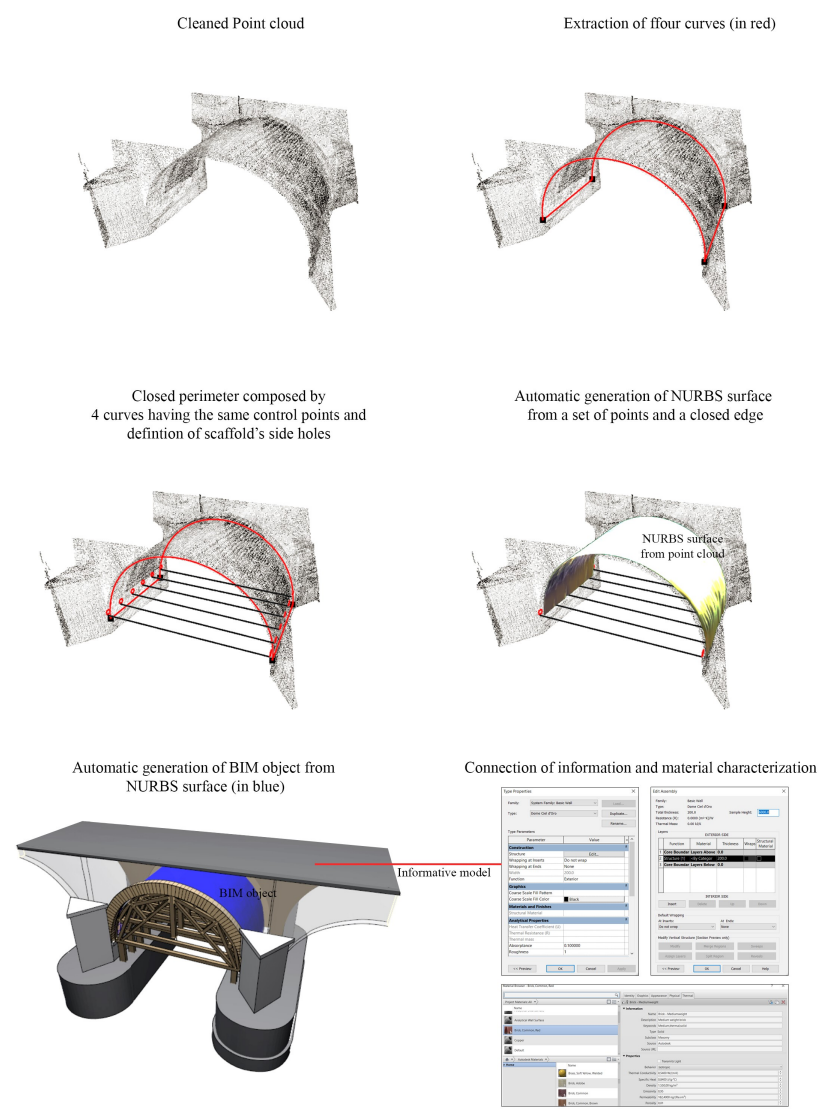

Figure 13. The research method: 'how to model a barrel vault by GOG 10'. Thanks to GOG 10, The surface is transformed into a parametric BIM object. The thickness of each vault of the bridge has been intercepted from the main two facades and the thickness of the arches which close the structure. 
The Groin vault (traditional modelling process) is also known as a double barrel vault. This type of structure, from a geometric point of view, can be considered as the intersection of two barrel vaults at the right angles. During the centuries, a groin vault provided less material and labour than a barrel vault where the load's thrust concentrated along the groins (four diagonal edges) formed by the two barrel's vaults intersection. The construction method was particularly used on the basement level and not used on the upper floors because it required great skill in cutting stone to form well-defined cross groins. For this reason, it replaced by the more flexible type of vaults such as the umbrella vault and the rib vault of gothic architecture in the later middle ages. Its surface is therefore constituted, in its simplest form, by a framework of four perimeter arches passing through the vault's centre. The centre is closed by a keystone or pyramid-shaped stone allowing the structure to self-supporting, unloading its weight on the perimeter supports such as walls, columns or pillars. Unlike simple vaults, the modeller must know a large number of commands oriented both to create surfaces and to subtract portions. Consequently, knowledge of cutting commands and the boolean difference must be added to the steps identified for the generation of a barrel vault.

The following figure shows, in addition to the steps related to the barrel vault, the main transformations to be performed for the correct generation of a groin vault.

As with the traditional geometric construction of simple vaults, the following procedure is not able to generate an irregular groin vault (Fig. 14). Moreover, the limited number of modelling tools in BIM applications further reduces the generation of complex shapes, exponentially limiting the generation of complex vaults corresponding to the point clouds.
The Groin vault by GOG 10 - As anticipated, the groin vault is considered to be a double barrel insertion. Unlike the dome vault, however, only the groins and the edge at the base are been considered for its generation. Each edge of this vault is crucial to the modelling. They are comprised of two diagonal arches that culminate at the top of the vault and four lines at the bottom of the structure. Morphological characteristics of the complex vaulted systems, in recent years, have required the creation of a large number of slices for the generation of HBIM models. The slicing, as already pointed out several times, required long lead times for the creation of reference planes and cross sections to achieve the geometric primitives of the selected artefact. The modelling of groin vault, the ribbed vault and umbrella vault, unlike simple vaults, are generated by geometrically complex intrados surfaces, based on irregular geometric primitives (Fig. 15).

Taking into consideration the generative principle of a groin vault where the intersecting of the rectangular-plan cross vault is clear as to obtain an adequate level of detail the portions of the vault (lunettes) can be entirely realised where they do not present geometric discontinuities. For this reason, the proposed method is based on the interpolation function based on the management of the NURBS surface through one external edge (contour line) and a great number of internal points, avoiding the generation of internal B-splines. Modelling result was ensured accurate surfaces within a limited computation of knot vectors, weight vectors, and one control network for each type of complex vaults. This factor has led to not considering the traditional generative technique, which involves long procedures not able to embed the complexity of irregular structures instead of GOG 10.
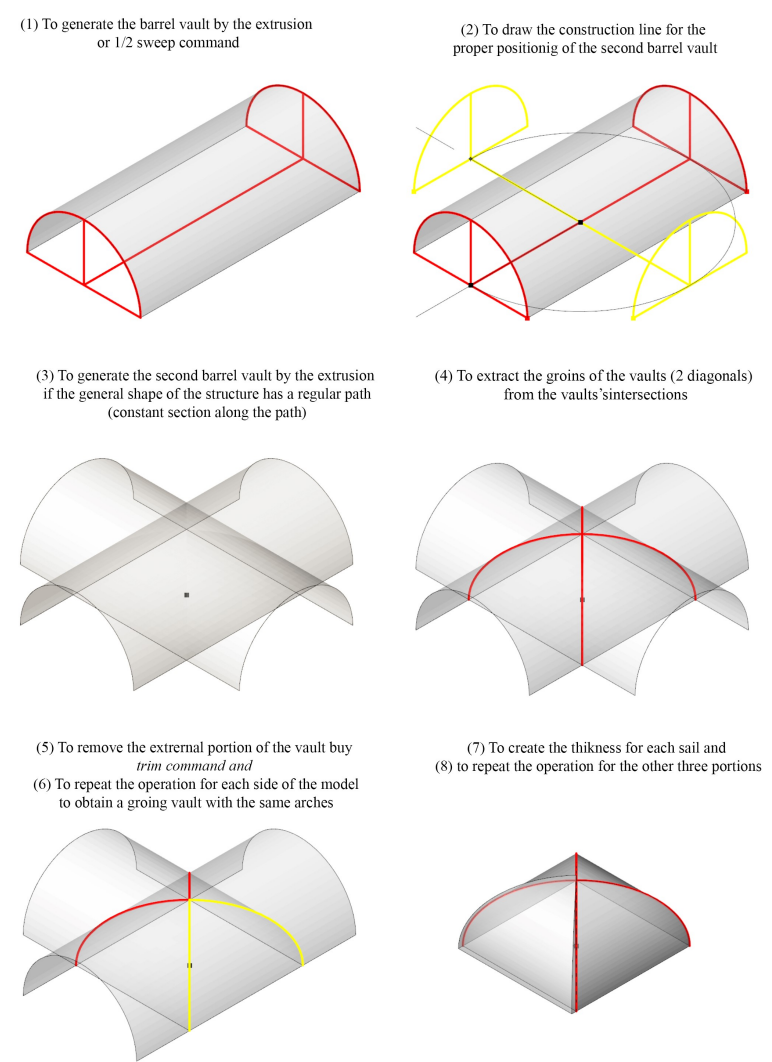

Figure 14. The traditional process: how to model a groin vault. The traditional process requires it is not able to consider structural deformations and geometric irregularities
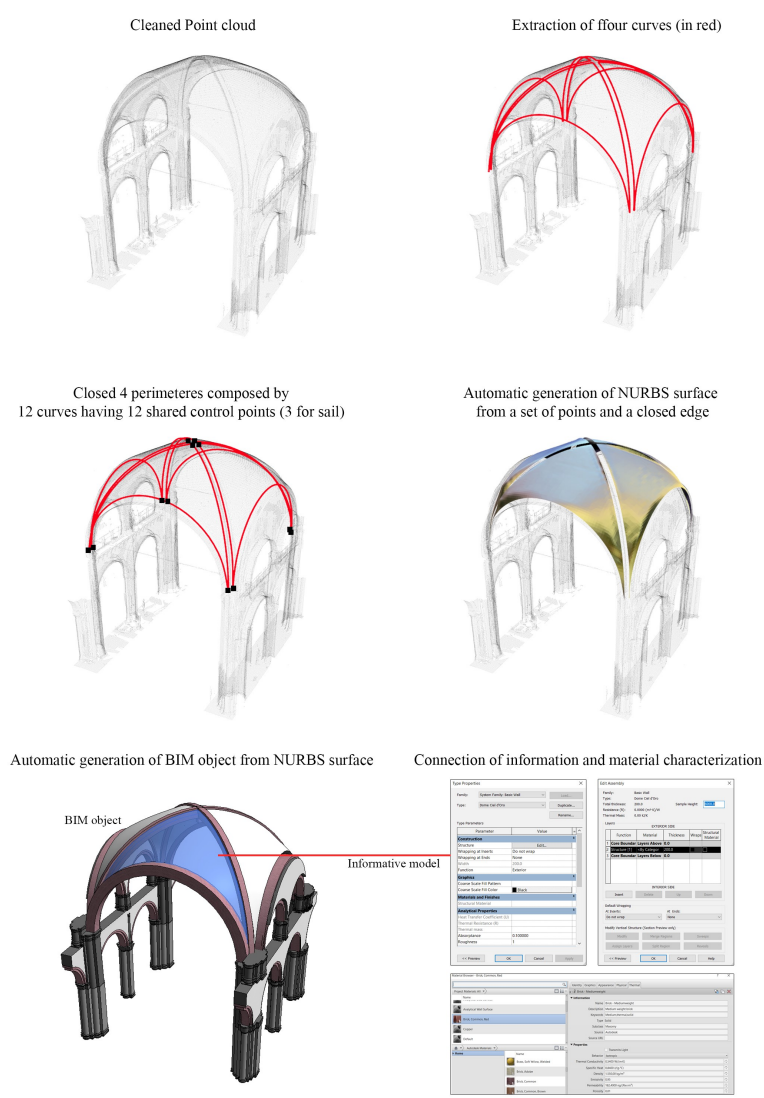

Figure 15. The research method: how to model a groin vault by GOG 10. It improves the generative process, simplifying the modelling for beginners and BIM experts at the same time. 


\section{DATA PROCESSING TIME AND THE AUTOMATIC VERIFICATION SYSTEM (AVS) OF THE GRADE OF ACCURACY (GOA) FOR VAULT MODELS}

The previous paragraph showed how GOG 10 appears to be a valid tool to improve the generation of very detailed complex architectural elements from point cloud data. Global processing time can be subdivided as follows:

- generation of geometric primitives with NURBS curves: 5 min (manually); 3 s (automatically);

- automated generation of the NURBS surfaces, including curves as boundaries and the point cloud as a constraint for interpolation: less than $3 \mathrm{~s}$;

- the geometric parametrisation of the thickness with the multilayer object: processing time less than $2 \mathrm{~s}$ (the user must only select the object and assign a specific family);

- inclusion of attributes: this part depends on the information available for each specific object (such as materials, physical and mechanical properties).

As a consequence, before carrying out the transformation from NURBS to BIM object, the analysis of the surfaces has been analysed in order to improve a system able to certify the quality of the model. For this reason, thanks to the analysis of the surface's curvature, an automatic verification system (AVS) has been identified to calculate the GOA and accurately provide the value of the standard deviation between NURBS surface and 3D scan. Point set deviation has been identified as the most suitable type of analysis of the proposed AVS in order to evaluate the GOA of the model between the point cloud and NURBS surfaces. A quantitative value is provided to quantify the quality of the models produced directly from the acquired point cloud (Fig. 16). This provides numerical values that represent the standard deviation between point clouds and the whole model without to define parameters analysis. This solution is not only an overall indication, but it can be reused to improve model quality over time and, thanks to its direct computation, it results in vast time savings.

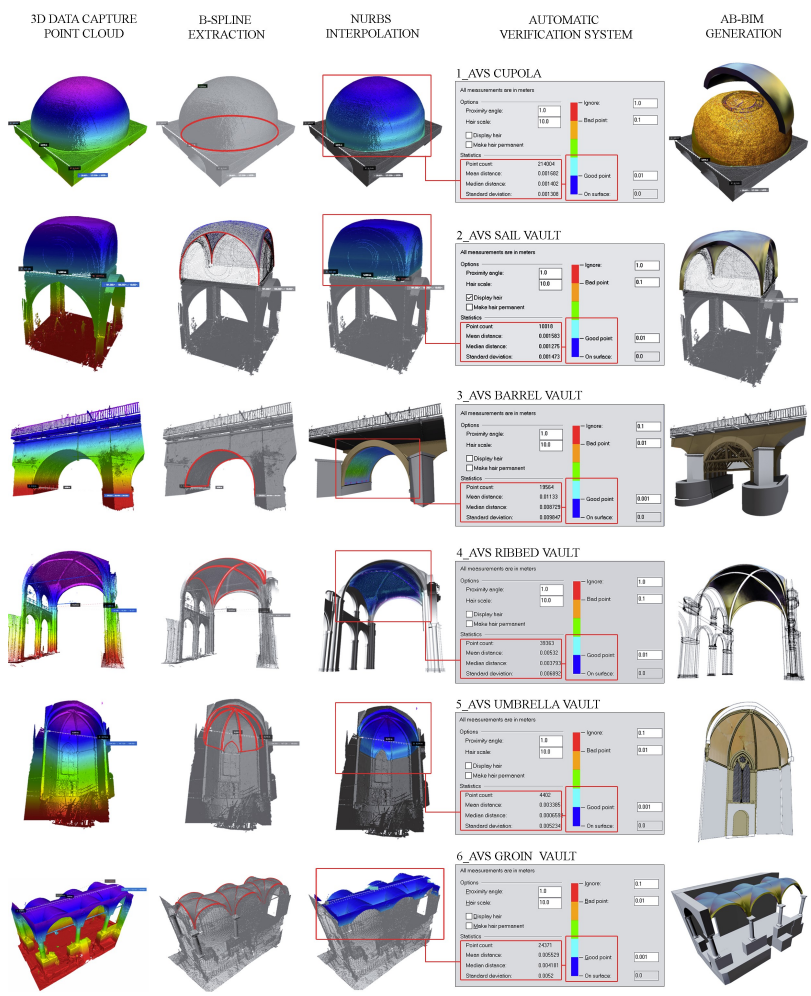

Figure 16. Thanks to GOG 10 and the add-in for Autodesk Revit users to get a GOA of $1 \mathrm{~mm}$ for complex structures.

\section{CONCLUSION}

The research method introduces novel modelling requirements with the aim of increasing automation in the generative process of HBIM models with a high grade of accuracy, supporting the information mapping and the information sharing at the same time. International standardisation experiences such as - PAS 1192 parts 1-2-3-4-5 (Great Britain); - COBIM- Common BIM Requirements- 2012 (Finland); - Statsbygg BIM Manual 1.2.1 (Norway); - AIA Document E201-2013 Project Digital Data Protocol Form and AIA Document E203-2013 Building Information Modelling and Digital Data Exhibit (USA) have identified the pros and cons for the management of heritage buildings. Compared to international systems, the UNI 11337 standard is introducing new guidelines and LOD for restoration. This innovative aspect is a step forward compared to the criteria for qualifying the built heritage in other countries. On the other hand, this study tries to support, through new grades of generation, accuracy and information (GOG, GOA), the construction of 'smart' models able to represent the reality detected, opening a reflection on different experiences made in the geomatics and restoration fields.

\section{REFERENCES}

Banfi, F., 2017: BIM orientation: grades of generation and information for different type of analysis and management process, Int. Arch. Photogramm. Remote Sens. Spatial Inf. Sci., XLII-2/W5, 57-64, doi.org/10.5194/isprs-archives-XLII-2-W5-57-2017

Banfi F., 2019: The integration of a scan-to-HBIM process in BIM application: the development of an add-in to guide users in Autodesk Revit, Int. Arch. Photogramm. Remote Sens. Spatial Inf. Sci., XLII-2/W11, 141-148, doi.org/10.5194/isprs-archives-XLII-2W11-141-2019.

Brumana, R., Condoleo, P., Grimoldi, A., Banfi, F., Landi, A. G., \& Previtali, M., 2018: HR LOD based HBIM to detect influences on geometry and shape by stereotomic construction techniques of brick vaults. Applied Geomatics, 10(4), 529-543.

Della Torre, S., 2015: Shaping tools for built heritage conservation: from architectural design to program and management: learning from 'Distretti culturali'.Community Involvement in Heritage, Garant 93-102.

Dore, C. \& Murphy, M., 2017: Current state of the art historic building information modelling. Int. Arch. Photogramm. Remote Sens. Spatial Inf. Sci. XLII-2/W5, 2017, doi:10.5194/ispr-archivesXLII-2-W5-185-2017.

Fai, S., \& Sydor, M., 2013: Building Information Modelling and the documentation of architectural heritage: Between the 'typical' and the 'specific'.2013 Digital Heritage International Congress (DigitalHeritage) IEEE., (Vol. 1, pp. 731-734).

Ioannides, M., \& Quak, E., 2014: 3D research challenges in cultural heritage. Lecture notes in computer science, 8355, 151.

Georgopoulos, A., 2018: Contemporary Digital Technologies at the Service of Cultural Heritage. Heritage Preservation. Springer, Singapore,. 1-20.

Remondino, F., \& Stylianidis, E., 2016: 3D recording, documentation and management of cultural heritage (Vol. 2). Whittles Publishing.

Tucci, G., \& Bonora, V., 2011: Geomatic techniques and 3D modelling for the survey of the Church of the Holy Sepulchre in Jerusalem. Proceedings XXIII CIPA Symposium, Prague, Czech Republic (Vol. 12, p. 16) 